\title{
Interaction between Players during a Sequence of Small-Sided Soccer Games: Effect of the Game Compartment
}

\author{
Kais Ghattassi ${ }^{*}$, Ahmed Graja ${ }^{1}$, Jamel Halouani ${ }^{2}$, Mohamed Amine Bouzid ${ }^{2}$ \\ ${ }^{1}$ Research Laboratory “Sport Performance Optimization”, CNMSS, Tunis, Tunisia \\ ${ }^{2}$ Unité de recherché "Education, Motricité, Sport et Santé, UR15JS01”, High Institute of Sports and Physical Education, University \\ of Sfax, Sfax, Tunisia \\ Email: *Kaisgatassi@yahoo.fr
}

How to cite this paper: Ghattassi, K., Graja, A., Halouani, J., \& Bouzid, M. A. (2017). Interaction between Players during a Sequence of Small-Sided Soccer Games: Effect of the Game Compartment. Advances in Physical Education, 7, 399-408. https://doi.org/10.4236/ape.2017.74033

Received: July 28, 2017

Accepted: October 31, 2017

Published: November 3, 2017

Copyright (c) 2017 by authors and Scientific Research Publishing Inc. This work is licensed under the Creative Commons Attribution International License (CC BY 4.0).

http://creativecommons.org/licenses/by/4.0/

\begin{abstract}
It is well known that the interaction between athletes has a positive effect on performance. In a sequence of small-sided games, soccer players must analyze their interactions (interaction between forwards and defenders) to adjust their position and engage in the creation of opportunities to score a goal. The aim of this study was to investigate the effect of interaction or relationship practices between players in football (forwards and defenders) through soccer small-sided games (SSG). Eighty soccer players (18.2 \pm 1.01 years, $1.80 \pm 0.05$ $\mathrm{m}$ and $75.0 \pm 7.6 \mathrm{~kg}$; mean $\pm \mathrm{SD}$ ) were volunteered to perform two sessions for four minutes with a recovery period of two minutes in a football court measuring $35 \times 30 \mathrm{~m}$ with small-sided games. Participants were divided in eight groups and each team was composed of five players with three defenders and two forwards $(3 \mathrm{D}+2 \mathrm{~F})$ for the first team, and three forwards and two defenders $(3 \mathrm{~F}+2 \mathrm{D})$ for the second team. The first session consists of playing the two teams against each other whereas the second session consists of playing the forwards against the defenders (5F vs. 5D). In each session, numbers of passes were recorded using video analysis. Additionally, interaction between players was obtained with sociometry questionnaire. The main results showed a significant difference of the interaction in favour of the same player's compartment compared to the players of different compartment $(p<0.05)$. However, the players of the same compartment emit the greatest number of passes and goals $(p<0.05)$. In conclusion, it's effective during the sequences of soccer SSG to play groups of the same compartment. This homogeneity in the compartments may allow better performance during football matches.
\end{abstract}

\section{Keywords}

Interaction, Soccer, SSG, Team-Mates, Performance 


\section{Introduction}

The psychosocial environment, or team climate as it is more commonly termed in sport, is comprised of the quantity, quality, and sequence of the interactions that occur among all team members (athletes and coaches included) (Carron et al., 2002). These interactions create a specific team atmosphere, which is responsible for much of the influence exerted on group members' behavior (Miles et al., 2011). Although, there is no attempt to deny that successful team performance is one major responsibility of the coach, athletes are also responsible for creating a team climate that emphasizes acceptance, understanding, and communication among other things (Sullivan \& Feltz, 2003). They should therefore become more sensitive and understand more clearly the relationships between themselves (Caperchione et al., 2011). Sport researchers interested in the quality of the sport experience have implemented a variety of strategies to address their concerns. Descriptive-analytic research focusing on the behavioral assessment and analysis of behaviors in athlete-athlete interaction settings is typified by the studies of Heuzé et al. (2006). Moreover, the interaction analysis strategy is to observe, describe, and analyze the quantity and quality of the interactions occurring in the sport environment for a specific period of time in game (Patterson et al., 2005). Otherwise, of the various paths to evaluate group environments in soccer, SSG is a most recent and promising approach to analyze interactions between athletes and other psychosocial characteristics (Kiritz \& Moos, 1974). Indeed, players must coordinate their actions with others across many different spatial and temporal scales (Cashmore, 2002). For example, soccer teammates on an offensive attack must coordinate their more immediate movements in order to complete a pass, while on a longer scale adjust their position and heading to create opportunities to score a goal (Hindmarsh \& Heath, 2000). On the other side, defenders must anticipate and match the offense's forwards and movements, while at the same time making subtle adjustments to steer their opponents to unfavorable positions, thereby reducing the threat of a score (Turman, 2005). In particular, in defensive play, they must make their teammates continually aware of potential problems regarding their own and the other team's locations and activities on court in ways that are often more time-pressured and less dependent on practiced game plans than is the case for attacking play in order to promote successful collaborative action that counteracts the opposition's attack (Lecouteur \& Foe, 2011).

In the same context, Trandafirescu (2015) have suggested that during a game of football, for example, players must focus on the movement of the ball, the movement and location of their own opposition player, and their teammates. Moreover, because of their continuous movement, the configuration of players on the court does not remain static at any point during the game players can be situated side-by-side, face-to-face or back-to-back. It is arguably easier, then, in many other complex interactive environments to establish mutual orientation and call a colleague into action, because the activities and objects that can: 1) 
constitute colleagues' shared orientation, and 2) form the basis of collaborative activity, are situated within the immediate vicinity typically in front of them (Lecouteur \& Foe, 2011).

SSG are one of the most common drills used by coaches for soccer training. Although in the past, SSG were mainly used to improve the interaction among players and to develop technical and tactical abilities, they are now employed by many amateur and professional teams as an effective tool for aerobic training (Rampinini et al., 2007).

However, few studies in the sport psychology literature, have examined the nature of actual interaction or relationship practices between players in football as they occur during play. There is therefore limited understanding of how successful communication is achieved between team members in real-life sports encounters. This article reports results from a study designed to address this gap in knowledge through soccer SSG between defenders and forwards players.

\section{Methodology}

\subsection{Participants}

Eighty junior soccer players $(18.2 \pm 1.01$ years, $1.80 \pm 0.05 \mathrm{~m}$ and $75.0 \pm 7.6 \mathrm{~kg}$; mean \pm SD) of 6 teams from the Tunisian league 1participated in this study. Experimental protocol of the study was made at the beginning of the season between august 2016 and October 2016. All of the players were fully informed about the procedures to be used and the experimental risks. Written informed consent was obtained by all players. All study procedures were approved by the general research ethics review board at the researchers' home university and by the football club's executive. Moreover, the participants were recruited based on: 1) they trained, 4 - 6 days per week for an average of $2 \mathrm{~h}$ daily in addition to the weekend match, and 2) they had at least 8 years of training experience. A clinical interview was carried out to verify the absence of exclusion criteria. These criteria were: heart failure and lower-limb injuries or muscle soreness during the experiment.

\subsection{Experimental Design}

The research protocol proposed in the study consisted to divided players in two teams as equitably as possible to avoid favoring any particular group or players. This was done to eliminate any impact or interference on the outcome of the game. Each team consisted of five players with three defenders and two forwards for the first team (3D: D1, D2 and D3 + 2F: F4 and F5) (Defender steam: DF) and three forwards and two defenders for the second team (3F: F1, F2 and F3 + 2D: D4 and D5) (Forwards team: FT). The second session consists of playing for wards vs. Defenders 5F vs. 5D). All players performed two sessions of SSG of four minutes with a passive recovery period of two minutes in a constant pitch dimensions (i.e., $30 \times 35 \mathrm{~m}$ ) but they have been further familiarized with the specific forms of SSG during two weeks before the experiment. These dimen- 
sions are standards' values and are used in anterior studies. On the other hand, they have been proposed to enhance soccer specific endurance capacity.

\subsection{Instruments}

\section{- The observation with video}

The two sessions of game are filmed with a digital camera (Sony Cyber Shot 14.1 megapixels). The video recordings were used to count the number of passes between players.

We opted for the choice of video recordings that to investigate the interaction effects of or relationship practices between players in football as they occur during play. In this context that interaction between players could affect performance. Moreover, video analysis used in the present study were chosen based scientific works focusing on didactic performances specific to soccer players (Mrayeh et al., 2014). Nevertheless, we think that this protocol we used could achieve, at least in part, the goal of this study.

So in choosing the means to collect the necessary study that we conducted we opted for the choice of video recordings that could bring every detail and provided a workable at all times memory. To carry out this research we relied on a didactic support, we crafted from some grids specialists (Bouthier, 1986) to facilitate note taking and help limit the parameters to be observed).

\section{- Sociometry method}

Players were exposed to a question session with duration of five minutes. The players were asked to answer the survey proposed by Moreno (1951) with sociometric method and its instrument (sociometric surveys) conducted a survey on the development and organization of the group, and the position of individuals in groups. The survey was used after its translation and calculated its reproducibility in our experimental. We realized two sessions before the experiment in order to check the reproducibility of the test results. The results of these methods are used to improve relationships in these groups, where possible, by regular and professional approach to improve the cohesion and unity of the group, as well as the adaptation and integration of "isolated" individuals. Questions in sociometric instrument on which players had to answer were:

- Who would you like to work with? (Positive choice)

- Who do you think would like to work with you? (Positive perception)

- With whom would not you like to work? (Negative choice)

- Who do you think would not like to work with you? (Negative perception)

In order to determine the functional status of individual players in the team, the following choice was formulated: respondents to indicate five players with whom they would prefer to play in the lineup, in order, from best to worst. On that occasion it was determined number of points from sociometric matrix, the number of mutual choice, and the number of times someone was chosen.

\subsection{Statistical Analyses}

Data are presented as mean and standard deviation. We used the comparison of 
statistical tools such as the means and variances for the two samples. Student test is a variance analysis tool 2 factors was used. To compare the interaction between defenders and forwards, a mixed design was established (time factor for testing: pre-post: repeated measurements) $\times 2$ (groups of factors: defenders and forwards). In addition, an analysis of variance in a factor with repeated measures was performed for each group to show changes pre and post-test in each group. We accepted $p \leq 0.05$ as a criterion for statistical significance. The statistical treatment was performed using the computer software (SPSS 21) operating on PC.

\section{Results}

\subsection{Influence of Interaction on Performance during Play}

The first step in the analysis and consideration of interpersonal relationships in the team is the video analysis. In the first condition, statistical analysis showed that the number of passes in DT between defenders was higher in comparison with the number of passes between defenders and forwards $(p<0.035)$. On the other hand, in the second condition, the number of passes between (D1, D2 and D3 is lower in comparison with the number passes between the same players in the first condition $(p<0.022)$ (Table 1$)$.

In addition, statistical analysis showed that the number of passes in FT between the forwards was higher in comparison with the number of passes between forwards and defenders $(p<0.041)$. On the other hand, in the second condition, the number of passes between the former forwards (F1, F2 and F3) is lower in comparison with the number of passes between the same players in the first condition $(p<0.022)$.

\subsection{Interactions between Defenders and Forwards}

Statistical analysis showed a higher scores of positive perception and positive choice between D1, D2 and D3 as compared to forwards (F4 and F5), while negative choice and negative perception scores were higher between forwards (F4 and F5) as compared to defenders (D1, D2 and D3) $(p<0.041)$. Additionally, our results showed a significant increase of positive perception, positive choice,

Table 1. Number of passes between players.

\begin{tabular}{cccc}
\hline Condition & \multicolumn{2}{c}{ Number of passes } & Number of goals \\
\hline \multirow{2}{*}{ Condition 1 } & D-D: $25 \pm 2.1^{\star}$ & F-F: $24 \pm 1.8^{*}$ & G1: $4 \pm 0.21$ \\
& D-F: $10 \pm 1.74^{\#}$ & F-D: $7 \pm 0.33^{\dagger}$ & G2: $7 \pm 0.41$ \\
Condition 2 & D-D: $17 \pm 1.24$ & F-F: $12 \pm 0.84$ & G1: $4 \pm 0.05$ \\
Total & D-N $: 18 \pm 2.04$ & F-N $: 19 \pm 1.12$ & G2: $11 \pm 0.7$ \\
& $70 \pm 1.78$ & $62 \pm 1.02$ & $26 \pm 0.34$ \\
\hline
\end{tabular}

*: significant difference in comparison with Condition 2 ; ": significant difference with D-D in the Condition $1 ;{ }^{\dagger}$ : significant difference with F-F in the Condition $1 ; \mathrm{N}^{\mathrm{D}}$ : two defenders playing in Condition $1 ; \mathrm{N}^{\mathrm{F}}$ : two forwards playing in Condition 1. 
negative choice and negative perception scores of D1, D2 and D3 in condition 1 as compared to condition $2(p<0.041)$ (Table 2$)$.

The Moreno sociogram showed positive interactions only between defenders and negative interactions between defenders and forwards (Figure 1). However, in the second condition we noted positive interactions between former defenders (D1, D2 and D3) and between old and new defenders (D1, D2, D3, D4 and D5) (Figure 2).

Similarly, Statistical analysis showed a higher scores of positive perception and positive choice between F1, F2 and F3 as compared to defenders (D4 and D5), while negative choice and negative perception scores were higher between defenders (D4 and D5) as compared to forwards (F1, F2 and F3) $(p<0.022)$. In addition, our results showed a significant increase of positive perception, positive choice, negative choice and negative perception scores between F1, F2 and F3 in condition 1 as compared to condition $2(p<0.041)$ (Table 3 ).

The Moreno sociogram showed positive interactions only between defenders and negative interactions between defenders and forwards (Figure 3). However, in the second condition we noted positive interactions between former defenders (F1, F2 and F3) and between old and new defenders (F1, F2, F3, F4 and F5) (Figure 4).

\section{Discussion}

The present study aimed to investigate the effect of interaction or relationship practices between players in football (forwards and defenders) through soccer

Table 2. Scores of choice and perception of defenders in comparison with partners.

\begin{tabular}{ccccccc}
\hline & & $\begin{array}{c}\text { Positive } \\
\text { choice }\end{array}$ & $\begin{array}{c}\text { Negative } \\
\text { choice }\end{array}$ & $\begin{array}{c}\text { Positive } \\
\text { perception }\end{array}$ & $\begin{array}{c}\text { Negative } \\
\text { perception }\end{array}$ & $\begin{array}{c}\text { Total } \\
\text { weight }\end{array}$ \\
\hline $\begin{array}{c}\text { Condition } \\
1\end{array}$ & $\mathrm{D} 1,2,3$ & $1.9 \pm 0.5$ & $1.2 \pm 0.6$ & $1.5 \pm 0.6$ & $1.2 \pm 0.4$ & $5.8 \pm 0.4$ \\
$\begin{array}{c}\text { Condition } \\
2\end{array}$ & $\mathrm{D} 4,5$ & $0.7 \pm 0.2^{*}$ & $2.5 \pm 0.3^{*}$ & $0.6 \pm 0.2^{*}$ & $2.1 \pm 0.5^{*}$ & $5.9 \pm 0.5$ \\
& $\mathrm{D} 4,5$ & $1.7 \pm 0.2$ & $0.1 \pm 0.3$ & $1.6 \pm 0.2$ & $0.1 \pm 0.4$ & $3.5 \pm 0.2$ \\
\hline
\end{tabular}

*: significant difference between two condition with D1, 2, $3(p<0.041)$; *: significant difference between D1, 2, 3 and F4, 5 in Condition $1(p<0.041)$.

Table 3. Scores of choice and perception of forwards in comparison with partners.

\begin{tabular}{ccccccc}
\hline & & $\begin{array}{c}\text { Positive } \\
\text { choice }\end{array}$ & $\begin{array}{c}\text { Negative } \\
\text { choice }\end{array}$ & $\begin{array}{c}\text { Positive } \\
\text { perception }\end{array}$ & $\begin{array}{c}\text { Negative } \\
\text { perception }\end{array}$ & $\begin{array}{c}\text { Total } \\
\text { weight }\end{array}$ \\
\hline $\begin{array}{c}\text { Condition } \\
1\end{array}$ & $\mathrm{~F} 1,2,3$ & $1.7 \pm 0.3$ & $1.4 \pm 0.5$ & $1.7 \pm 0.3$ & $1.4 \pm 0.1$ & $6.2 \pm 0.9$ \\
& $\mathrm{D} 4,5$ & $0.5 \pm 0.1^{*}$ & $2.4 \pm 0.2^{*}$ & $0.4 \pm 0.1^{*}$ & $2.4 \pm 0.2^{\star}$ & $5.7 \pm 0.4$ \\
$\begin{array}{c}\text { Condition } \\
2\end{array}$ & $\mathrm{~F} 1,2,3$ & $3.4 \pm 0.1^{\star}$ & $0.2 \pm 0.3^{*}$ & $3.4 \pm 0.1^{\star}$ & $0.1 \pm 0.5^{\star}$ & $7.1 \pm 0.6^{*}$ \\
\hline
\end{tabular}

*: significant difference between two condition with F1, 2, $3(p<0.022)$; ${ }^{*}$ significant difference between F1, 2, 3 and D4, 5 in Condition $1(p<0.041)$. 


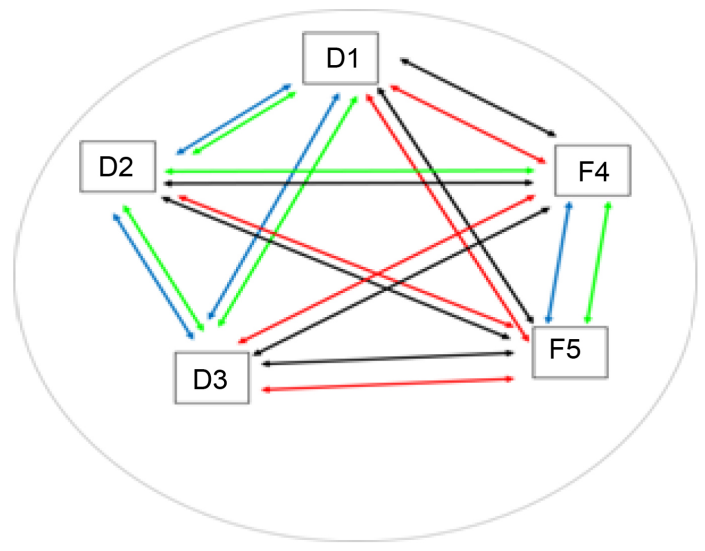

Figure 1. Interaction between forwards and defenders. Green arrow: positive choice; Blue arrow: positive perception; Red arrow: negative choice; Black arrow: negative perception.

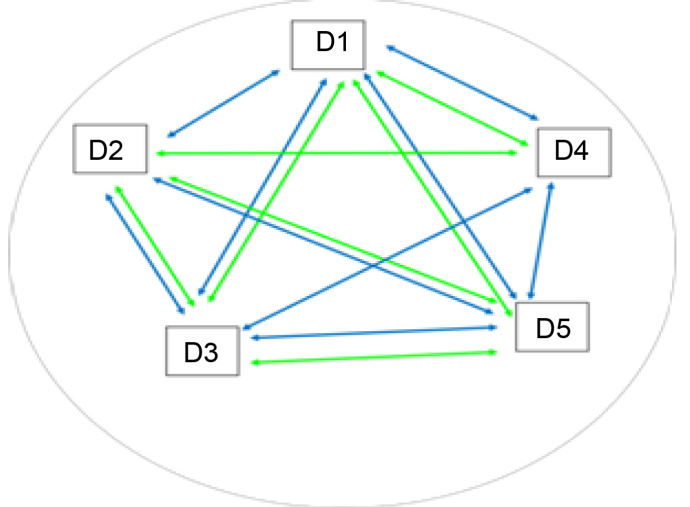

Figure 2. Interaction between defenders. Green arrow: positive choice; Blue arrow: positive perception; Red arrow: negative choice; Black arrow: negative perception.

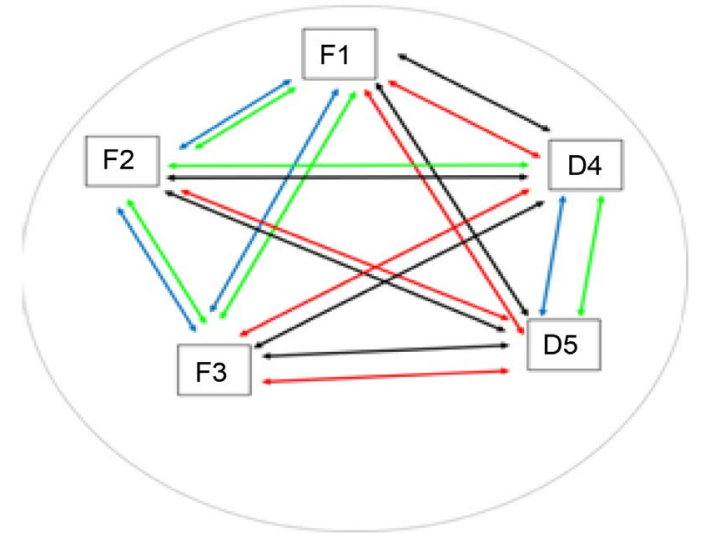

Figure 3. Interaction between forwards and defenders. Green arrow: positive choice; Blue arrow: positive perception; Red arrow: negative choice. Black arrow: negative perception. 


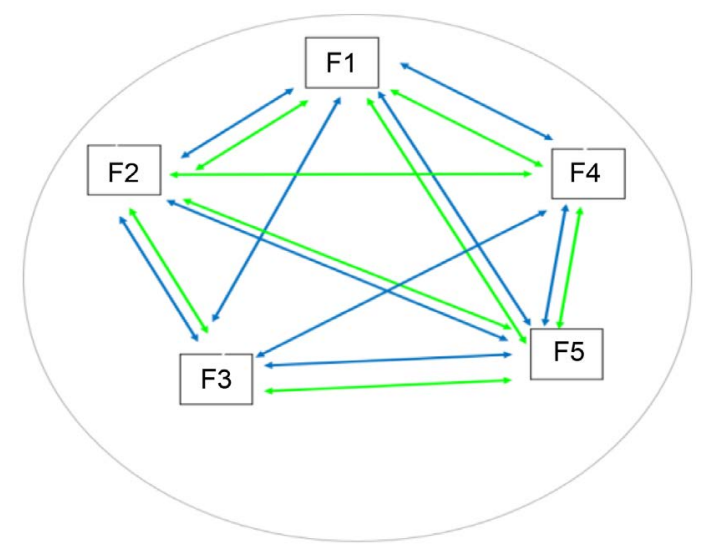

Figure 4. Interaction between forwards. Green arrow: positive choice; Blue arrow: positive perception; Red arrow: negative choice; Black arrow: negative perception.

SSG. The main findings showed a difference between players of same compartment compared to the players of different compartment. However the players of the same compartment have recorded number of passes and of goals more importantly.

Soccer game makes not only physical, but also pedagogical and psychological influence on players. The individual approach to a player in matches is not limited to only the acquisition of technical or tactical skills. During a sports season especially when players do not yet know one another well enough, it is important to ensure their cooperation (Bailey, 2006). It is not enough for a player just to meet with other players; they should be put into the circumstances that would allow communication and interaction skills acquisition and development (Plaude, 2010).

In fact, the results obtained in this study revealed significant difference in favour to the same player's compartment as compared to the players of different compartments.These findings are in accordance with the results of Carron et al. (2002) who have shown important interaction between players of football.

Additionally, our results showed that the players of the same compartment emit the greatest number of passes and goals. Our results are in line with those of Trandafirescu (2015), who revealed that Rugby players of the same compartment emit more passes between them than players from different compartments. This could explain, at least in part, that match of football may be considered as a complex activity, the basis of which: the cooperation between players (Bailey, 2006). In other words, a player should understand the primary task and secondary ones, control his/her own behavior for discussing match's result, helping his/her teammates and evaluating problem situations, and solving potentially arising interpersonal conflicts in the process of interaction (Dale \& Wrisberg, 1996; Sullivan \& Feltz, 2003). In the same context, participating in soccer SSG aimed at interaction skills development, the players were learning to listen to one another, to talk politely, to help one another, as well as to consider success 
and failure of their activity, to correct their own and others' mistakes, to observe the activity of the other players, to overcome difficulties. Thus, soccer SSG and group work help players to develop interaction and cooperation, taking initiative, solving conflicts, setting the aim of work, mutual assistance and self-criticism (Dombrovskisa et al., 2014).

On the other hand, team members must monitor each other's conduct whilst engaged in individual activities. In particular, in defensive play, they must make their teammates continually aware of potential problems regarding their own and the other team's locations and activities on court in ways that are often more time-pressured and less dependent on practiced game plans than is the case for attacking play in order to promote successful collaborative action that counteracts the opposition's attack.

To the best of the authors' knowledge, this is the first study to explore the effect of interaction or relationship practices between players in football (forwards and defenders) through soccer SSG. However, some limitations inherent to the experimental protocol of this study warrant mention. First, the sample size was small. Secondly, we have not used any other teams.

It seems that the interaction between athletes has a positive effect on performance. The homogeneity in the compartments allows us to obtain the best performance during football matches. As the sampling of players is not big, it is necessary to broaden the study using not only players from same teams, but also from other teams. In the future studies, it is also necessary to consider age or experience differences and to make a comparative analysis of control and experimental group results.

\section{References}

Bailey, R. (2006). Physical Education and Sport in Schools: A Review of Benefits and Outcomes. International Journal of Health Sciences, 76, 397-401. https://doi.org/10.1111/j.1746-1561.2006.00132.x

Bouthier, D. (1986). Comparaison expérimentale des effets de différents modèles didactiques des sports collectifs. [Experimental Comparison of the Effects of Different Didactic Models in Team Sports.] In EPS, contenus et didactique (pp. 85-89). Paris: SNEP.

Caperchione, C., Mummery, W. K, \& Duncan, M. (2011). Investigating the Relationship between Leader Behaviours and Group Cohesion within Women's Walking Groups. Journal of Science and Medicine in Sport, 14, 325-330.

https://doi.org/10.1016/j.jsams.2011.03.005

Carron, M., Colman, J., \& Wheeler, D. (2002). Cohesion and Performance in Sport: A Meta-Analysis. Journal of Sport and Exercise Psychology, 24, 168-188. https://doi.org/10.1123/jsep.24.2.168

Cashmore, E. (2002). Sport and Exercise Psychology: The Key Concepts (2nd ed.). London: Routledge.

Dale, G. A., \& Wrisberg, C. A. (1996). The Use of a Performance Profile Technique in a Team Setting: Getting the Athletes and Coach on the "Same Page". The Sport Psychologist, 10, 261-277. https://doi.org/10.1123/tsp.10.3.261 
Dombrovskisa, V., Gusevaa, S., \& Capulis, S. (2014). Cooperation and Learning Effectiveness of First Graders during Sports Lessons. Social and Behavioral Sciences, 112, 124-132.

Heuzé, J. P., Raimbault, N., \& Fontayne, P. (2006). Relationships between Cohesion, Collective Efficacy, and Performance in Professional Basketball Teams: An Examination of Mediating Effects. Journal of Sports Sciences, 24, 59-68.

https://doi.org/10.1080/02640410500127736

Hindmarsh, J., \& Heath, C. (2000). Embodied Reference: A Study of Deixis in Workplace Interaction. Journal of Pragmatics, 32, 1855-1878. https://doi.org/10.1016/S0378-2166(99)00122-8

Kiritz, S., \& Moos, R. H. (1974). Physiological Effects of Social Environments. Psychosomatic Medicine, 36, 96-114. https://doi.org/10.1097/00006842-197403000-00002

Le Couteur, A., \& Feo, R. (2011). Real-Time Communication during Play: Analysis of Team-Mates' Talk and Interaction. Psychology of Sport and Exercise, 12, 124-134.

Miles, J. et al. (2011). Do Birds of a Feather Move Together? Group Membership and Behavioral Synchrony. Experimental Brain Research, 211, 495-503. https://doi.org/10.1007/s00221-011-2641-z

Moreno, J. L. (1951). Sociometry: Experimental Method and the Science of Society. Beacon House.

Mrayeh, M., Hawani, A., \& Mechrgui, A. (2014). Compared Efficiency of Abilities to Steer Learning Situations by Two Categories of Sport and Physical Education Teachers Having Different Levels of Academic Qualification. Advances in Physical Education, 4, 149-163. https://doi.org/10.4236/ape.2014.43019

Patterson, M. M., Carron A. V., \& Loughead, T. M. (2005). The Influence of Team Norms on the Cohesion-Self-Reported Performance Relationship: A Multi-Level Analysis. Psychology of Sport and Exercise, 6, 479-493.

Plaude, I. (2010). Kooperatīvāmācīšanās. [Co-Operative Learning.] Rīga: RaKa.

Rampinini, E. et al. (2007). Factors Influencing Physiological Responses to Small-Sided Soccer Games. Journal Sports of Sciences, 25, 659-666. https://doi.org/10.1080/02640410600811858

Sullivan, P., \& Feltz, D. (2003). The Preliminary Development of the Scale for Effective Communication in Team Sports. Journal of Applied Sport Psychology, 33, 1693-1715.

Trandafirescu, G. (2015). Particular Aspects Concerning Cohesion of the Athlete Group at Football Teams. Social and Behavioral Sciences, 180, 1369-1373.

Turman, P. D. (2005). Coaches' Use of Anticipatory and Counterfactual Regret Messages during Competition. Journal of Applied Communication Research, 33, 116-138. https://doi.org/10.1080/00909880500045072 